\title{
Unusual clinical picture in an overweight patient with Legionella pneumophila: A case report
}

\author{
Leonidas Grigorakos*1,2, Daria Lazarescu², Anthi Georgiadou², Maria Bikou ${ }^{3}$, Magda Gkouni $^{3}$, Yorgos Lafazanos ${ }^{3}$, \\ Charalambos Roumbeas ${ }^{3}$, Nikolaos Markou ${ }^{2}$ \\ ${ }^{1}$ Faculty of Nursing, National and Kapodistrian University of Athens, Greece \\ ${ }^{2}$ Intensive Care, "KAT" Trauma Hospital of Athens, Kifissia, Athens, Greece \\ ${ }^{3}$ Intensive Care and Pneumonology, "Athina" General Clinic, Athens, Greece
}

Received: March 30, 2017

DOI: $10.5430 /$ crim.v4n3p31
Accepted: June 18, 2017

Online Published: July 3, 2017

URL: https://doi.org/10.5430/crim.v4n3p31

\begin{abstract}
This case report describes a case of a patient with Legionnaires' disease (LD) manifested three days upon his return from a medical conference, which took place in a hotel close to the seaside. Our patient presented to the hospital febrile, weak, confused and with mild difficulty in breathing. After being subjected to several tests, he was diagnosed with LD. Even though his initial urinary antigen test (UAT) was negative, subsequent immunofluorescent assays (IFA) were positive for Legionella pneumophila (LP). The patient was immediately initiated specific antibiotics therapy and supportive measures. After 11 days he was released from the hospital with considerable melioration of his clinical condition and with specific instructions to continue therapy at home.
\end{abstract}

Key Words: Legionnaires' disease, Air condition, Infectious diseases, Diagnosis, Overweight patient

\section{INTRODUCTION}

Legionnaires' disease (LD) is a form of bacterial pneumonia caused by Legionella species. Legionella is transmitted by inhalation of aerosolized contaminated water drops and it leads to LD after an incubation period of 2-10 days. ${ }^{[1]}$ Given that the course of disease may be characterized as extremely severe, a high proportion of patients usually necessitate intensive care. While the overall mortality rate is $15 \%$, healthcare associated cases usually have a higher rate, which may reach even 30\%. ${ }^{[2,3]}$ Legionnaires' infection may exhibit a variety of symptoms involving the respiratory, gastrointenstinal and neurologic system. Most Legionella infections, for which a source can be established, are related to contaminated manmade aquatic environments (cooling towers, whirlpools and warm water systems). ${ }^{[1]}$ However, for most LD cases, the source of infection remains unknown. ${ }^{[4]}$ One of the explanations for these unsolved investigations may be the broad range of (relatively rare) potential sources of infection that are involved in LD but not always considered during source investigations. $^{[4]}$ In a recent review, it was presented an overview of all confirmed and potential sources of Legionella to optimize source investigations and unravel the contribution of these sources to LD burden. ${ }^{[4]}$ In addition to the well-known sources such as cooling towers and whirlpools, relatively rare sources such as ice machines, milling and mist machines have also been previously described as confirmed source of infection. ${ }^{[5]}$ In this case report, we describe an LD patient with late response to therapy, who presented to our hospital with fever, weakness, confusion and mild difficulty in breathing.

\footnotetext{
*Correspondence: Leonidas Grigorakos; Email: grigorakos@ parliament.gr; Address: Faculty of Nursing, National and Kapodistrian University of Athens, Intensive Care, "KAT” Trauma Hospital of Athens, Kifissia, 2 Nikis St, 14561, Athens, Greece.
} 


\section{CASE PResentation}

A 39-year-old man, bio-pathologist, was admitted to our hospital with the primary symptoms of fever and confusion. The patient was overweight but his medical history was unremarkable and he did not use any drugs. Three days before admission, upon the patient's return from a medical conference, which took place in a hotel close to the seaside, he had developed fever, cough, discomfort and dyspnea. On the day of admission (Day 1), the patient's wife noticed that he could not stand by himself and he was febrile, despite common antipyretics administered.

On admission, the patient was restless and had a temperature of $40.1^{\circ} \mathrm{C}$, the patient's blood pressure was $100 / 75 \mathrm{mmHg}$, and pulse rate was 102 beats/min His oxygen saturation was $89 \%$. The patient's arterial blood gases (ABGs) were: $\mathrm{PaO}_{2} 69 \mathrm{mmHg}$, $\mathrm{PCO}_{2} 36 \mathrm{mmHg}, \mathrm{pH} 7.42, \mathrm{HCO}_{3} 22 \mathrm{mEq} / \mathrm{L}$. Laboratory tests revealed a normal white blood cell (WBC) count but detected neutrophilia (WBC count, $8.36 \times 10^{3} / \mu \mathrm{L}$, of which neutrophils $96.5 \%$ ), hyponatremia $(\mathrm{Na}, 128 \mathrm{mEq} / \mathrm{L})$, a markedly increased creatine kinase $(\mathrm{CK})$ (1,252 IU/L), and increased fibrinogen/fibrin degradation products (FDP) $(21.3 \mu \mathrm{g} / \mathrm{ml})$. Pulmonary auscultation revealed signs of crackles, while chest radiography revealed consolidation of the lower right lobe. Repeat sputum specimens (up to $5 \mathrm{ml}$ each) were collected but no bacilli were detected. Patient's respiratory distress was addressed with oxygen through Venturi mask and aerosol therapy. At the same time, antipyretic and empiric antibiotic therapy were initiated, while continued hydration was ensured.

On Day 2, a Legionella urinary antigen test (UAT) was performed and it was negative for LP. Despite the negative test result, the suspicion of LD remained and a new UAT for LP was performed on new urine samples. This second test showed a weak positive result after 30 minutes of incubation.

Thus, immunofluorescent assays (IFA) serotypes 1-8 were performed. In short, serum dilutions were reacted into acetone-fixed LP serogroup 1 for 30-immunoglobulin $\mathrm{G}$ (IgG) and polyimmunoglobulin (pIgR)- or 90immunoglobulin $\mathrm{M}(\operatorname{IgM})$ and immunoglobulin $\mathrm{A}(\operatorname{Ig} \mathrm{A})$ minutes. Through a sorbent treatment in the $\operatorname{IgM}$ and $\operatorname{IgA}$ determinations we ought to exclude interferences from IgG and rheumatoid factor. We incubated the fluorescein isothiocyanate (FITC)-specific conjugates for a period of $30 \mathrm{~min}$. As the $\operatorname{IgG}$ or pIgR titers were higher than $1 / 256$ and the IgM or IgA titers were higher than $1 / 96$, they were considered positive for LP. Once diagnosis revealed, intravenous Erythromycin $(1,000 \mathrm{mg} / 8 \mathrm{~h})$ was initiated. This specific antibiotic was preferred to the detriment of others (i.e. Lev- ofloxacin or Azitrhomycin) as we took into consideration the clinical picture of our patient but also due to the fact that, from the very beginning, we had reasonable suspicions and indications that he was infected by LP.

The patient's clinical condition gradually improved and he was released from our clinic on day 11 , with specific guidelines for outpatient therapy. Specifically, the attending physician prescribed antibiotics therapy at home, with orally administered Clarithromycin $(500 \mathrm{mg}$ ) every 8 hours, for better concentration due to patient's body overweight, initially for 14 days. Physician also prescribed IFA screening every 7 days and computed tomography (CT) of the lungs was performed after one month. The first IFA screening was still positive for LP, even though the levels of LD antibodies were considerably reduced. More so, the patient remained extremely weak and he was having a late response to the therapy. Thus, antibiotics therapy was prolonged for one more month. One month after, he was also subjected to lung CT, which did not reveal any pathological abnormalities. After two months of repeated IFA screening, results were negative for LP and so, antibiotics therapy was brought to an end.

\section{Discussion}

Most LD cases in Europe are LP serogroup 1-related. ${ }^{[6]}$ Nevertheless, diagnosing LD can put a serious challenge to medical personnel because it is usually very difficult to differentiate it from other types of pneumonia.

The phenotypic methods currently used are urinary antigen detection, serum antibody titration and culture. Among these, the most common method of laboratory diagnosis is the urinary antigen which can be traced in the chronic phase of disease through the enzyme immunoassay (EIA). Nevertheless, this method has proved to have an importantly decreased sensitivity in particular cases. ${ }^{[7]}$ Thus, detection of LD through serology titration of Legionella-specific antibody response by an immunofluorescent antigen (IFA) test or an enzymelinked immunosorbent assay (ELISA)-based test is usually preferred, even though serological cross-reactions with other micro-organisms have been cited. ${ }^{[8-10]}$

In our case, the results of the diagnostic test performed demonstrate that the diagnosis of LD should not be rejected after a negative UAT. In cases of suspected LD, and a negative UAT, urine test should be repeated with an expanded time of the urine sampling. ${ }^{[11]}$ More so, diagnostic should be expanded with serology tools, such as IFA.

To differentiate Legionella infection -through clinical, radiologic or analytical characteristics- from other causes of pneumonia, may pose a great challenge. Thus, in several cases, antimicrobial agents which are efficient against LP should 
however be used in the therapy for both community-acquired and nosocomial pneumonia. The most common antibiotic for LD's therapy has proved to be the Erythromycin. However, in cases of patients with severe illness or immunosuppression and confirmed LD, a combined therapeutic approach with Rifadin is usually more efficient. ${ }^{[12]}$

In our case, due to unremarkable medical history, but also taking into consideration Rifadin's probable side effect of liver toxicity, we decided to avoid therapy with this specific drug and, after intravenous treatment with Erythromycin, we prescribed to our patient orally administered Clarithromycin.

Besides Legionella, there are several other causative agents for community-acquired pneumonia that are often found among travelers. In a previous study on causes of pneumonia among travelers, a wide range of causative agents were reported (Streptococcus pneumoniae, Mycoplasma pneumoniae, Coxiella burnetti, Leptospira sp., Mycobacterium tuberculosis), including LP. ${ }^{[13]}$ This indicates that diagnosis in returning travelers with pneumonia requires an open mind with respect to the etiological spectrum of involved pathogens.
For LD, an estimated $20 \%$ of all reported European cases are travel associated which should encourage awareness among physicians to consider this causative agent. ${ }^{[14]}$ In this respect, we identified the hotel in which the medical conference took place and we urged hotel's management to take the necessary measures in order to limit a possible outbreak of LD.

In conclusion, diagnosis and treatment of LD should be targeted in cases of patients at increased risk for illness and complications due to Legionella infection. In this sense, in order to diagnose LP pneumonia, serology is still a valuable tool. Moreover, physicians should keep a high suspicion of LD in cases in which they observe an obvious disproportional image between not particularly severe chest radiography findings and an extremely weak clinical picture of the patient. Furthermore, despite correct treatment applied, patient may have a slow recovery. In our case, this slow response seems to have been caused by the fact that our patient was overweight.

\section{CONFLicts OF INTEREST Disclosure}

The authors have declared no conflicts of interest.

\section{REFERENCES}

[1] Fields BS, Benson RF, Besser RE. Legionella and Legionnaires' disease: 25 years of investigation. Clin Microbiol Rev. 2002; 15: 506-26. PMid:12097254 https ://doi.org/10.1128/CMR.15.3.506-5 26.2002

[2] Mandell LA, Wunderink RG, Anzueto A, et al. Infectious Diseases Society of America/American Thoracic Society consensus guidelines on the management of community-acquired pneumonia in adults. Clin Infect Dis. 2007; 44(suppl 2): S27-72. PMid:17278083 https://doi.org/10.1086/511159

[3] US Environmental Protection Agency (EPA). Basic information about disinfectants in drinking water: Chloramine, chlorine and chlorine dioxide. Available from: http://water.epa.gov/drink/ contaminants/basicinformation/disinfectants.cfm. Accessed 20 March 2017.

[4] Van Heijnsbergen E, Schalk JA, Euser SM, et al. Confirmed and potential sources of Legionella reviewed. Environ Sci Technol. 2015; 49: 4797-815. PMid:25774976 https : //doi .org/10.1021/acs . est. $5 \mathrm{~b} 00142$

[5] Euser S, Diederen B, Bakker M, et al. Legionnaires' disease after a campervan holiday: a case report. J Travel Med. 2016; 23(1): 34-5. PMid:26782124 https://doi.org/10.1093/jtm/tav004

[6] Joseph C. Surveillance of Legionnaires' disease in Europe. In Legionella: Proceedings of the 5th International Conference on Legionella. 2003: 311-7. Edited by Marre R, Abu Kwaik Y, Bartlett C, et al. Washington, DC: American Society for Microbiology.
[7] Helbig JH, Uldum SA, Bernander S, et al. Clinical utility of urinary antigen detection for diagnosis of community-acquired, travelassociated, and nosocomial legionnaires' disease. J Clin Microbiol 2003; 41: 838-40. PMid:12574296 https ://doi.org/10.1128/ JCM. 41 . 2.838-840. 2003

[8] Wilkinson HW, Cruce DD, Broome CV. Validation of Legionella pneumophila indirect immunofluorescence assay with epidemic sera. J Clin Microbiol. 1981; 13: 139-46. PMid:7007417

[9] Musso D, Raoult D. Serological cross-reactions between Coxiella burnetii and Legionella micdadei. Clin Diagn Lab Immunol. 1997; 4: 208-12. PMid:9067657

[10] Fallon RJ, Abraham WH. Crossreactions between Legionella and Campylobacter spp. Lancet. 1992; 340: 551-2.

[11] Bruin JP, Diederen BM. Evaluation of the Meridian TRU Legionella, a new rapid test for detection on Legionella pneumophila serogroup 1 antigen in urine samples. Eur J Clin Microbiol Infect Dis. 2013; 32: 333-4. PMid:23010904 https ://doi .org/10.1007/s10096 -012-1745-0

[12] Amsden GW. Treatment of Legionnaires' disease. Drugs. 2005; 65(5): 605-14. PMid:15748096 https://doi .org/10.2165/00003495 $-200565050-00003$

[13] Ansart S, Pajot O, Grivois JP, et al. Pneumonia among travelers returning from abroad. J Travel Med. 2004; 11: 87-91. PMid:15109472 https : //doi.org/10.2310/7060.2004.17055

[14] Beaute J, Zucs P, de Jong B. Legionnaires' disease in Europe 20092010. Euro Surveill. 2013; 18: 20417. PMid:23515061 